1 Giess R, Naumann M, Werner E, et al. Injections of botulinum toxin $\mathrm{A}$ into the salivary glands improve sialorrhoea in amyotrophic lateral sclerosis. $\mathcal{F}$ Neurol Neurochir Psy chiatry 2000;69:121-123.

2 Mezaki T, Kaji R, Kohara N, et al. Development of general weakness in a patient with amyotrophic lateral sclerosis after focal botulinum toxin injection. Neurology 1996;46: 845-6.

3 Bakheit AM, Ward CD, McLellanDL. Generalized botulism-like syndrome after intramuscular injections of botulinum toxin type A. A report of two cases. $\mathcal{F}$ Neurol Neurochir Psychia try 1997;62:196-200.

4 Erbguth F, Claus D, Engelhardt A, et al. Systemic effect of local botulinum toxin injections unmasks subclinical Lambert-Eaton myasthenic syndrome. 7 Neurol Neurochir Psychiatry 1993;56:1235-36.

5 Jost WH. Treatment of drooling in Parkinson's disease with botulinum toxin. Mov Disord 1999;14:1057.

\section{The authors reply:}

We appreciate the comments by Winterholler et al on our article ${ }^{1}$ on botulinum toxin (BTX/A) treatment of sialorrhoea in patients with amyotrophic lateral sclerosis (ALS). Although we did not find any serious side effects after transcutaneous injections of BTX/A into the parotid and submandibular glands Winterholler et al report on sublingual salivary gland infection in one patien and deterioration of dysphagia in another patient after a transductal approach. These complications support our notion that the individually tolerated dose of BTX/A in patients with ALS may be low and also indicate that the transcutaneous approach as performed in several studies ${ }^{1-3}$ may be safer than the retrograde transductal injection. This is not unexpected as the transductal approach has possibly a higher risk of infection because of the reduced salivary gland secretion rate found in patients with ALS. ${ }^{1}$ In addition, the total dose of $25 \mathrm{MU}$ Botox for the sublingual glands may be rather high in view of the close anatomical relation of these glands to the pharyngeal muscles. We therefore underscore our previous suggestion to start with injections of the parotid glands alone and to cautiously escalate the dose and number of injected glands. ${ }^{1}$ In view of the potential risk of BTX/A in deteriorating ALS symptoms injections should be restricted to otherwise intractable and extremely disabled patients with ALS who have sialorrhoea.

M NAUMANN R GIESS

K SCHWAGER

K V TOYKA

Department of Neurology and Department of Otorhinolaryngology,

Bayerische-fulius-Maximilians-Universität, fosef-Schneider-Strasse 11, 97080 Würzburg, Germany

Correspondence to: Dr M Naumann naumann@mail.uni-wuerzburg.de

1 Giess R, Naumann M, Werner E, et al. Injections of botulinum toxin $A$ into the salivary glands improve sialorrhoea in amyotrophic lateral sclerosis. $\mathcal{F}$ Neurol Neurosurg Psychiatry 2000;69:121-3.

2 Jost WH. Treatment of drooling in Parkinson's disease with botulinum toxin. Mov Disord 1999;14:1057.

3 Bhatia KP, Münchau A, Brown P. Botulinum toxin is a useful treatment in excessive drooling of saliva [letter]. I Neurol Neurosurg Psychiatry 1999;67:679.
Treatment of early onset Parkinson's disease with ropinirole

The recent editorial ${ }^{1}$ supporting initial treatment of early onset Parkinson's disease with a dopamine agonist hinged in part on the demonstration $^{2}$ in 268 patients that treatment of early onset Parkinson's disease with ropinirole alone or with supplementary levodopa/dopa decarboxylase inhibitor (benserazide) (LD/DDI) resulted in substantially less dyskinesia than with LD/DDI alone, with only slightly less motor benefit. Five per cent of patients on ropinirole alone developed dyskinesia after 5 years, compared with $25 \%$ with ropinirole plus LD/DDI, and $45 \%$ of those on LD/DDI alone). The trial design allowed LD/DDI supplementation if response was inadequate and additional trial drug could not be tolerated. Up to $24 \mathrm{mg}$ ropinirole and $1200 \mathrm{mg} \mathrm{LD} / \mathrm{DDI}$ daily were allowed. Sixty six per cent of patients completing the ropinirole arm required supplementation, the average mean daily dose of ropinirole at 5 years being $16.5 \mathrm{mg}$, compared with $753 \mathrm{mg}$ of LD/DDI when the second was used alone.

It is unfortunate that the study required a three times daily dosage regime. It seems possible that this accounts for the surprising $33 \%$ of patients on LD/DDI alone who withdrew as a result of early adverse events, and for the occurrence of nausea in $49.4 \%$ of patients on LD/DDI alone. Whether smaller, more frequent, dosage would have allowed better tolerance of and motor response to ropinirole, it is not surprising that frequent dyskinesia was seen at 5 years on three times daily dosage of LD/DDI. A substantial proportion of patients on LD/DDI (43.8\%) were also on selegiline, amplifying the effect substantially. By comparison, the 5 year study of immediate release (IR) and controlled release (CR) LD/DDI (carbidopa) in 681 patients, ironically reported earlier in 1997, and later in $1999,{ }^{3}$ in whom dosage could be adjusted up to five or more times a day resulted not only in a lower frequency of dyskinesia $(20.6 \% \mathrm{IR}, 21.7 \% \mathrm{CR})$ at 5 years but also a lower mean total daily dose $(426 \mathrm{mg}$ IR; 510 mg CR (bioequivalent)).

Whereas it may be argued that different drug preparations and methods of assessment invalidate comparison, it may be simply that less frequent higher pulsatile dosage provokes not only greater peak dose dyskinesia but also, as a mirroring effect, greater off time as postsynaptic mechanisms adapt to cope with surges of dopamine and perhaps lose sensitivity to troughs. Patients seen during troughs would be liable to have their dose increased. If the interdose interval were fixed this would lead to a vicious circle.

Given reports of long term resolution of dyskinesia and on/off effects in response to various methods of continuous stimulation, at an appropriate strength, including continuous daytime jejunal infusion of LD/DDI (with little or no change in $\mathrm{LD} / \mathrm{DDI}$ dosage requirement over 57 months), ${ }^{4}$ and of a neuroprotective effect of levodopa, ${ }^{5}$ the results of Rascol et $a l^{2}$ should not dissuade others from pursuing oral treatment with LD/DDI in a more frequent, lower dose regime. With gradual (allowing for the long duration action of levodopa) titration of slow release LD/DDI dosage and interdose interval (if necessary using a timer), against response and compliance of patient (or carer), it may in theory and, with sufficient observation and titration, in practice be possible to approximate to such a steady state stimulation and response. This would have potentially less risk for developing hallucinations, and would cost less.

J R PONSFORD

Department of Neurology, Walsgrave Hospital NHS Trust, Clifford Bridge Road, Coventry CV2 2DX, UK

1 Brooks DJ. Dopamine agonists: Their role in the treatment of Parkinson's disease [editorial]. $\mathcal{F}$ Neurol Neurosurg Psychiatry 2000;68:685-90.

Neurol Neurosurg Psychiatry 2000;68:685-90.
Rascol O, Brooks DJ, Korczyn AD, et al. A five year study of the incidence of dyskinesia in year study of the incidence of dyskinesia in
patients with early Parkinson's disease who patients with early Parkinson's disease who
were treated with ropinirole or levodopa. $N$ were treated with ropinirole
Engl f Med 2000;342:1484-91.

3 Koller WC, Hulton JT, Tolosa E, et al. Immediate release and controlled release carbidopa/ levodopa in Parkinson's disease. Neurology 1999;53:1012-19.

4 Syed N, Murphy J, Zimmermann T Jr, et al. Ten years experience with enteral levodopa infusions for motor fluctuations in Parkinson's disease. Mov Disord 1998;13:336-8.

5 Murer G, Dziewczapolski G, Menalled LB, et al. Chronic levodopa is not toxic for remaining dopamine neurons, but instead promotes their dopamine neurons, but instead promotes their lesions. Ann Neurol 1998;43:561-75.

Brooks replies:

Ponsford seems to focus primarily on the design and findings of the 056 trial of ropinirole versus levodopa in early Parkinson's disease recently reported in the $N$ Engl F $\mathrm{Med}^{1}$ rather than the editorial as a whole; however, to take up his points:

Firstly, he suggests that it is unfortunate that the 056 trial required a three times daily levodopa dosage regime as use of more frequent smaller doses could have reduced the incidence of dyskinesias. We chose a three times daily regime in part to match the three times daily regime of ropinirole and also because it was thought that this regime reflected common clinical practice in patients with early Parkinson's disease. A trial formally comparing use of multiple low doses of levodopa versus a three times daily medium dose regime in early Parkinson's disease would, however, be of great interest. It might well be that the multiple low dose approach in early disease would spare complications but this has yet to be shown. Addition of a catechol-O-methyltransferase inhibitor to smooth out the plasma levodopa profile in early Parkinson's disease might also prove beneficial.

Secondly, he suggests that the allowed presence of selegeline may have magnified the tendency of levodopa to cause complications. This could indeed be the case although stratifying for selegeline usage in the levodopa arm did not highlight any such effect.

Thirdly, he suggests that allowing the use of slow release levodopa preparations in early Parkinson's disease could have been beneficial. There is currently no trial data to support this viewpoint; on the contrary, early use of either slow release madopar or sinemet has been reported to be associated with a similar prevalence of complications as the use of standard preparations.

My current feeling is that early use of dopamine agonists in suitable patients with Parkinson's disease remains a reasonable strategy to delay complications. It may well be that in the future, however, a more optimal way of delivering levodopa is devised which achieves continuous and uniform dopaminergic stimulation and so reduces the prevalence of fluctuations and dyskinesias.

D J BROOKS Neurology Department, Imperial College School of Medicine, Hammersnith Hospital, London W12 ONN, UK 
1 Rascol O, Brooks DJ, Korczyn AD, et al. A five year study of the incidence of dyskinesia in patients with early Parkinson's disease who were treated with ropinirole or levodopa N Engl f Med 2000;342:1484-91.

\section{Does disturbed homocysteine and folate metabolism in depression result from enhanced oxidative stress?}

In their recent article, Bottiglieri et al describe increased homocysteine concomitant with decreased folate concentrations in a subgroup of patients with depression. In addition, some relation between reduced folate availability and disturbed monoamine metabolism was found. The close relation between increased homocysteine and reduced folate concentrations, which was described previously in other clinical conditions such as cardiovascular and cerebrovascular diseases is usually ascribed to a reduced dietary intake of folate, and dietary supplementation with folate is capable of reducing hyperhomocysteinaemia.

The coincidence described of disturbed homocysteine and monoamine metabolism may shed some additional light to the possible mechanism underlying this metabolic abnormality. Both metabolic pathways depend on the presence of reduced pteridine species: (1) the biosynthesis of methionine requires supply of methyl groups from methyl-5,6,7,8-tetrahydrofolic acid, deficiency of which results in hyperhomocysteinaemia; (2) biosynthesis of serotonin, dopamine, and noradrenaline (norepinephrine) depends on the presence of 5,6,7,8 tetrahydrobiopterin, deficiency of which thus results in monoamine deficiency. Both tetrahydropteridines are recycled by dihydropteridine reductases ${ }^{2}$; both compounds have strong reducing capacities and are thus rapidly oxidised by oxidising chemicals. Interestingly, recent studies show that depression is associated with activation of the immune system, ${ }^{3}$ and it is even speculated that an infectious agent might be involved. Immune system activation is accompanied by an increased production of reactive species by cytotoxic cells such as activated monocytes and macrophages to achieve antimicrobial and cytocidal activities. Activated human monocytes/macrophages also release increased amounts of neopterin - another pteridine derivative - which is a sensitive index for the mediation of cell mediated immune reactions in patients. ${ }^{4}$ Recent data also point to a new functional aspect of neopterinnamely, to enhance oxidative processes. Increased concentrations of neopterin have been described in patients with depression. Therefore the question arises whether oxidative stress rather than insufficient dietary intake of folate is the basis of 5,6,7,8-tetrahydrofolate and also 5,6,7,8tetrahydrobiopterin deficiency. Interestingly, in patients with Alzheimer's dementia a similar relation has already been demonstrated: hyperhomocysteinaemia was associated with reduced folate concentrations, but also an increased degree of immune activation could be detected in the same patients. ${ }^{5}$ There is good reason to think that the scenario might be similar in patients with depression, and enhanced oxidative stress due to chronic immune system activation is a major cause of the loss of reductants such as

5,6,7,8-tetrahydrofolic acid and 5,6,7,8tetrahydrobiopterin.

B WIDNER

D FUCHS

Institute of Medical Chemistry and Biochemistry, Fritz Pregl Strasse 3, A-6020 Innsbruck, Austria; University of Innsbruck, Ludwig Boltzmann Institute of AIDS-Research, Innsbruck, Austria

F LEBLHUBER

Department of Gerontology, Landesnervenklinik Wagner-fauregg, Linz, Austria B SPERNER-UNTERWEGER Department of Psychiatry, University of Innsbruck, Austria

Correspondence to: Dr D Fuchs

Dietmar.Fuchs@uibk.ac.at

1 Bottiglieri T, Laundy M, Crellin R, et al. Homocysteine, folate, methylation, and monoamine cysteine, folate, methylation, and monoamine
metabolism in depression. $\mathcal{f}$ Neurol Neurosurg metabolism in depression.

2 Armarego WLF. A 20 year association with pteridines: chemistry, biochemistry and molecular biology. Pteridines 1998;9:55-68.

3 Maes M, Bosmans E, Scharpe S, et al. Plasma soluble interleukin-2-receptors in depression: relationships to plasma neopterin and serum IL-2 concentrations and HPA-axis activity. Eur Psychiatry 1995;10:397-403.

4 Widner B, Baier-Bitterlich G, Wede I, et al. Neopterin: indicator of oxidative stress and part of the cytotoxic armature of activated part of the cytotoxic armature of activated 102.

5 Leblhuber F, Walli J, Artner-Dworzak E, et al. Hyperhiomocysteinemia in dementia. $\mathcal{F}$ Neural Hyperhiomocysteinemia in
Transm 2000:107;1069-74.

Reynolds and Botiglieri reply:

We thank Widner et al for suggesting an explanation of our findings of impaired folate and monoamine metabolism in some patients with depression.

The relation between homocysteine and folate is well established, which is why we included it in our study. We agree that simply dietary deficiency is an inadequate explanation for folate deficiency in many patients with depression as several studies have failed to confirm this. ${ }^{1}$ We have recently reported a fall in CSF folate with aging ${ }^{2}$ and this may be a factor contributing to the high incidence of folate deficiency in psychogeriatric patients, including depression and dementia. ${ }^{1}$ We have also reported a fall in CSF tetrahydrobiopterin (BH4) in depression which is correlated with folate deficiency, as reflected in a fall in red cell folate, and with impaired monoamine metabolism - that is, a fall in CSF monamine metabolites. $^{3}$

The mechanisms of these relations between impaired folate and monoamine metabolism remain uncertain but the suggestion that oxidative stress plays a part is speculative. We are unaware of any clinical or experimental evidence that oxidative stress leads to folate deficiency. It has been suggested that folates play a part in maintaining $\mathrm{BH} 4$ synthesis. ${ }^{34}$ The relation of folate and $\mathrm{BH} 4$ to aging requires clarification. It is also relevant that S-adenosyl-methionine, the major methyl donor in the brain which derives its methyl group from methyl folate can, like folates, increase the turnover of monoamines in the brain. ${ }^{5}$ This and other evidence suggest that methylation mechanisms are involved in these relations and in mood and cognitive function. ${ }^{6}$

E H REYNOLDS

Institute of Epileptology, Weston Education Centre, King's College, Denmark Hill Campus, Cutcombe Road, London SE5 9Pf, UK

T BOTTIGLIERI

Metabolic Disease Center, Baylor Research Institute, 3812 Elm Street, Dallas, Texas 75226, USA
Correspondence to: Dr E H Reynolds reynolds@buckles.u-net.com

1 Bottiglieri T, Crellin RF, Reynolds EH. Folate and neuropsychiatry. In: Bailey LB, ed. Folate in health and disease. New York: Marcel Dekker, 1995:435-62.

2 Bottiglieri T, Reynolds EH, Laundy MC Folate in CSF and age. $\mathcal{F}$ Neurol Neurosurg Psychiatry 2000;69:562.

3 Bottiglieri T, Hyland K, Laundy M, et al. Folate deficiency, biopterin and monoamine metabolism in depression. Psychol Med 1992;22:871-

4 Kaufman S. Some metabolic relationships between biopterin and folate:implications for the "methyl folate trap hypothesis". Neurochem Res 1991;16:1031-6.

5 Losada ME, Rubio MC. Acute effects of S-adenosyl-methionine on catacholaminergic central function. Eur f Pharmacol 1989;163: 353-6.

6 Reynolds EH, Carney MWP, Toone BK. Methylation and mood. Lancet 1984;ii:196-8.

\section{Long term follow up after} perimesencephalic subarachnoid haemorrhage

Marquardt et al describe the clinical course and long term outcome of 21 patients they diagnosed as having a perimesencephalic haemorrhage. ${ }^{1}$ The paper raises two questions.

The first is an impression of an "emperor's new clothes syndrome" given by the first figure of the publication. This figure shows a slice of the CT made shortly after the initial episode of headache in the patient reported to have a recurrent episode of perimesencephalic haemorrhage. The legend of the figure states that the CT shows extravasated blood in the perimesencephalic subarachnoid space, but we fail to see any blood at all. Thanks to the electronic availability of the fournal we were able to review not only the paper version of the figure, but also an enlarged version on screen. Even after enlargement no blood was seen; the slice nicely shows the tentorium adjacent to the ambient cisterns, the proximal parts of the posterior cerebral arteries, and perfectly clear CSF in the perimesencephalic (chiasmatic and partly the ambient and quadrigeminal) cisterns and in the frontal interhemispheric and sylvian fissures.

There are several explanations for this diagnostic mystery. Firstly, the authors may have submitted an inappropriate slice of the CT. In some patients with perimesencephalic haemorrhage, the prepontine cistern is the only site where CT shows blood. ${ }^{2}$ If blood was visible in the prepontine cistern in this particular patient, the authors have indeed found a patient with a perimesencephalic, non-aneurysmal haemorrhage with a recurrent haemorrhage. Given the unique character of this sequence of events, it would be fair to provide the appropriate slice to convince readers of the fournal.

Secondly, if no evidence of blood is found even in the prepontine cistern, the patient may have had a CT negative subarachnoid haemorrhage. In patients with ruptured aneurysms CT can be negative, even if performed within 12 hours after onset of the haemorrhage. ${ }^{3}$ But a negative CT plus a negative angiography does not add up to a diagnosis of perimesencephalic haemorrhage.

Thirdly, the patient may not have had a subarachnoid haemorrhage at all. The case report tells us that lumbar puncture was positive, but does not give details. Because 